\title{
An Efficient and Inexpensive Refrigerated LC System for H/D Exchange Mass Spectrometry
}

\author{
Theodore R. Keppel, ${ }^{1,2}$ Martin E. Jacques, ${ }^{1,4}$ Robert W. Young, ${ }^{3}$ Kenneth L. Ratzlaff, ${ }^{1,3}$ \\ David D. Weis ${ }^{1,2}$ \\ ${ }^{1}$ Department of Chemistry, University of Kansas, 1251 Wescoe Hall Drive, Lawrence, KS 66045, USA \\ ${ }^{2}$ The R. N. Adams Institute for Bioanalytical Chemistry, University of Kansas, Lawrence, KS USA \\ ${ }^{3}$ Instrumentation Design Laboratory, University of Kansas, Lawrence, KS, USA \\ ${ }^{4}$ Clinical Research Laboratories, Lenexa, KS, USA
}

\begin{abstract}
Loss of deuterium label during the LC step in amide hydrogen/deuterium exchange mass spectrometry $(\mathrm{H} / \mathrm{D}-\mathrm{MS})$ is minimized by maintaining an acidic mobile phase $\mathrm{pH}$ and low temperature $\left(\mathrm{pH} 2.5,0{ }^{\circ} \mathrm{C}\right)$. Here we detail the construction and performance of a low-cost, thermoelectrically refrigerated enclosure to house high-performance liquid chromatography (HPLC) components and cool mobile phases. Small volume heat exchangers rapidly decrease mobile phase temperature and keep the temperature stable to $\pm 0.2{ }^{\circ} \mathrm{C}$. Using a superficially porous reversed-phase column, we obtained excellent chromatographic performance in the separation of peptides with a median peak width of $4.4 \mathrm{~s}$. Average deuterium recovery was $80.2 \%$ with an average relative precision of $0.91 \%$.
\end{abstract}

Key words: H/D exchange, Back-exchange, Refrigeration, Amide hydrogen/deuterium exchange

\section{Introduction}

$\mathrm{H}$ ydrogen/deuterium exchange mass spectrometry (H/DMS) has become widespread in studying protein conformation and dynamics in solution [1]. Deuterium uptake information can be localized using digestion of deuterated proteins with acid-tolerant proteases followed by LC separation [2]. H/D exchange is initiated by dilution of protein into $\mathrm{D}_{2} \mathrm{O}$ and quenched by mixing samples with acid to decrease $\mathrm{pH}$ to $\sim 2.5$. Unfortunately, once the reaction has been quenched, the deuterium label slowly back-exchanges with solvent hydrogen [3]. Immediate and stable cooling is necessary to slow the rate of back-exchange during a short

Electronic supplementary material The online version of this article (doi:10.1007/s13361-011-0152-6) contains supplementary material, which is available to authorized users.

Correspondence to: David D.Weis; e-mail: dweis@ku.edu
LC run. A typical approach is to immerse all LC components in an ice/water [3] or temperature-controlled water bath [4]. Baths are inexpensive and offer efficient cooling of LC components. However, troubleshooting leaks is nearly impossible and water can damage submerged components. Baths also pose the risk of spills or leaks into the LC/MS. Hence, iceless cooling systems for refrigerated high-performance liquid chromatography (HPLC) have been developed for H/D-MS [5-7]. In our lab, conventional refrigerators had unacceptable variation in temperature, typically at $4 \pm 2{ }^{\circ} \mathrm{C}$, and long re-equilibration when solvent reservoirs were refilled. Furthermore, even with forced-air convection, cooling is inefficient since heat must be transferred from the mobile phase to the LC components and then dissipated into the air. A system featuring direct mobile phase cooling using thermoelectrically-cooled mobile phase heat exchangers could only reach $4{ }^{\circ} \mathrm{C}$ and suffered from long gradient delays [7]. A thermal chamber to cool the HPLC components to $1.25{ }^{\circ} \mathrm{C}$ has been reported, but little 
Table 1. Deuterium Recovery for Deuterated Peptides

\begin{tabular}{|c|c|c|c|c|c|c|c|c|}
\hline \multirow[t]{2}{*}{ Peptide $^{a}$} & \multirow[t]{2}{*}{ Sequence } & \multirow{2}{*}{$\frac{\begin{array}{c}\text { Average } \\
\text { deuterated mass }\end{array}}{(\mathrm{Da})^{\mathrm{b}}}$} & \multirow{2}{*}{$\begin{array}{c}\text { Theoretical } \\
\text { undeuterated mass } \\
\text { (Da) }\end{array}$} & \multirow{2}{*}{$\begin{array}{c}\text { Mass Increase } \\
(\mathrm{Da})^{\mathrm{c}}\end{array}$} & \multirow{2}{*}{$\begin{array}{l}\text { Precision } \\
\operatorname{RSD}(\%)^{\mathrm{d}}\end{array}$} & \multirow{2}{*}{$\begin{array}{c}\text { Exchangeable } \\
\text { amide } \mathrm{H}^{\mathrm{e}}\end{array}$} & \multirow{2}{*}{$\begin{array}{c}\text { Deuteration } \\
(\%)\end{array}$} & \multirow{2}{*}{$\frac{\text { Peak width }^{\mathrm{f}}}{(\mathrm{s})}$} \\
\hline & & & & & & & & \\
\hline $1-7$ & GLSDGEW & $766.82 \pm 0.05$ & 762.76 & 4.06 & 1.2 & 5 & 85.4 & $3.56 \pm 0.03$ \\
\hline $8-13$ & QQVLNV & $703.51 \pm 0.02$ & 699.80 & 3.71 & 0.66 & 4 & 97.7 & $3.76 \pm 0.03$ \\
\hline $8-29$ & QQVLNVWGKVEADIAGHGQEVL & $2407.27 \pm 0.16$ & 2390.65 & 16.62 & 0.93 & 20 & 87.5 & $5.78 \pm 0.25$ \\
\hline $14-29$ & WGKVEADIAGHGQEVL & $1719.69 \pm 0.10$ & 1708.87 & 10.82 & 0.94 & 14 & 81.3 & $4.38 \pm 0.06$ \\
\hline $56-69$ & KASEDLKKHGTVVL & $1533.16 \pm 0.10$ & 1524.76 & 8.40 & 1.2 & 12 & 73.7 & $7.48 \pm 0.54$ \\
\hline $84-96$ & AELKPLAQSHATK & $1400.95 \pm 0.08$ & 1393.59 & 7.36 & 1.0 & 10 & 77.5 & $4.44 \pm 0.16$ \\
\hline $110-134$ & AIIHVLHSKHPGDFGADAQGAMTKA & $2586.11 \pm 0.12$ & 2572.90 & 13.21 & 0.88 & 22 & 63.2 & $4.18 \pm 0.28$ \\
\hline $125-139$ & ADAQGAMTKALELFR & $1631.09 \pm 0.07$ & 1621.86 & 9.23 & 0.77 & 13 & 74.7 & $6.12 \pm 0.31$ \\
\hline $137-153$ & LFRNDIAAKYKELGFQG & $1981.75 \pm 0.08$ & 1970.23 & 11.52 & 0.67 & 15 & 80.9 & $4.56 \pm 0.12$ \\
\hline Mean & & & & & 0.91 & & 80.2 & \\
\hline Angiotensin $\mathrm{I}^{\mathrm{g}}$ & DRVYIHPFHL & $1301.24 \pm 0.02$ & 1296.48 & 4.76 & 0.34 & 7 & 71.7 & \\
\hline Angiotensin $\mathrm{I}^{\mathrm{h}}$ & DRVYIHPFHL & $1301.16 \pm 0.02$ & 1296.48 & 4.69 & 0.44 & 7 & 70.5 & \\
\hline
\end{tabular}

${ }^{\text {a }}$ Peptides with residue numbers are from myoglobin.

${ }^{b}$ Mean value of 3 replicates \pm standard deviation.

${ }^{\mathrm{c} D}$ Deuterated mass-undeuterated mass.

${ }^{\mathrm{d}}$ Relative standard deviation (\%) calculated as standard deviation of the average deuterated mass divided by mass increase.

${ }^{\mathrm{e}}$ For a peptide with a free N-terminus, the number of exchangeable amides $=$ number of residues - number of prolines not in the first two residues -2 .

${ }^{f}$ Chromatographic peak full width at half-maximum, mean value of three replicates \pm standard deviation.

${ }^{\mathrm{g}}$ Angiotensin I with $0{ }^{\circ} \mathrm{C}$ protease column.

${ }^{\mathrm{h}}$ Angiotensin I with $15^{\circ} \mathrm{C}$ protease column.

detail was provided [5]. A ultra performance liquid chromatography (UHPLC) system, designed specifically for the Waters nanoAcquity, has also been reported but requires specialized LC pumps and bulky water chillers for heat dissipation [6].

Here, we detail the performance of a compact "all-in-one" refrigerated unit. The system features an internal protease column oven to enhance digestion efficiency. The unit is constructed from off-the-shelf components, requires minimal specialized assembly, and has a small footprint. A novel single valve design simplifies sample injection and HPLC separation procedures. We also provide an empirical demonstration of cooling efficiency, chromatographic performance, and deuterium recovery.

\section{Experimental}

\section{Temperature Measurements}

To protect the thermocouple from solvent damage, neat water was used for all temperature measurements. Under our conditions, the differences in the thermal properties of different LC solvents are negligible (see Supplementary Material). Fluid temperature was measured by diverting flow through a short piece of PEEK tubing at various points. An insulated thermocouple was fed into the open end of the PEEK tubing. Water from this deliberate leak was collected in a beaker. Air temperature was measured with a bare junction thermocouple. Both thermocouples were calibrated against melting ice using a single-point offset correction. Additional details are in the Supplementary Material.

\section{H/D Exchange}

To ensure complete deuteration, peptic peptides were prepared by digesting myoglobin with pepsin before labeling [8]. Five $\mu \mathrm{L}$ aliquots of myoglobin peptides or angiotensin I were deuterated with $95 \mu \mathrm{L}$ of deuteration buffer $(10 \mathrm{mM}$ phosphate, $50 \mathrm{mM}$ sodium chloride, $\mathrm{pH}$ 6.9). Exchange was quenched after at least $89 \mathrm{~min}$ with $100 \mu \mathrm{L}$ of ice-cold $200 \mathrm{mM}$ sodium phosphate (pH 2.6). To maximize reproducibility, quenched samples were loaded into the $100 \mu \mathrm{L}$ sample loop (Fig. 1) $60 \mathrm{~s}$ after quench followed by sample injection after an additional 30 s. Nineteen-fold dilution with $\mathrm{D}_{2} \mathrm{O}$ gives a maximum possible deuteration of $95 \%$. Our deuteration values were normalized as a percentage of this maximum (Table 1). Fully deuterated angiotensin I was prepared as described above except it was not digested with pepsin.

A detailed description of the construction of the refrigerated enclosure and all other experimental details can be found in the Supplementary Material.

\section{Results and Discussion}

\section{Description of the Refrigerated System}

Back-exchange is minimized when LC conditions are maintained at the lowest possible temperature. To meet this need, we constructed a thermoelectrically cooled (i.e., Peltier effect) compartment with precise mobile phase and independent protease column temperature controls (see Fig. 1). The enclosure is cooled using an air-to-air thermoelectric cooler module. Mobile phases are cooled by heat exchangers $(2 \mu \mathrm{L}$ internal volume) mounted to a thermoelectric cold plate assembly. Heat is dissipated to the surroundings by forced air convection, eliminating the need for secondary heat transfer to a circulating chiller [6]. Since proteolysis can be more efficient at elevated temperatures [6], we incorporated an insulated, low power oven $(<5 \mathrm{~W})$ so that the protease column can be warmed up to $20^{\circ} \mathrm{C}$ above the enclosure temperature. Temperatures are 


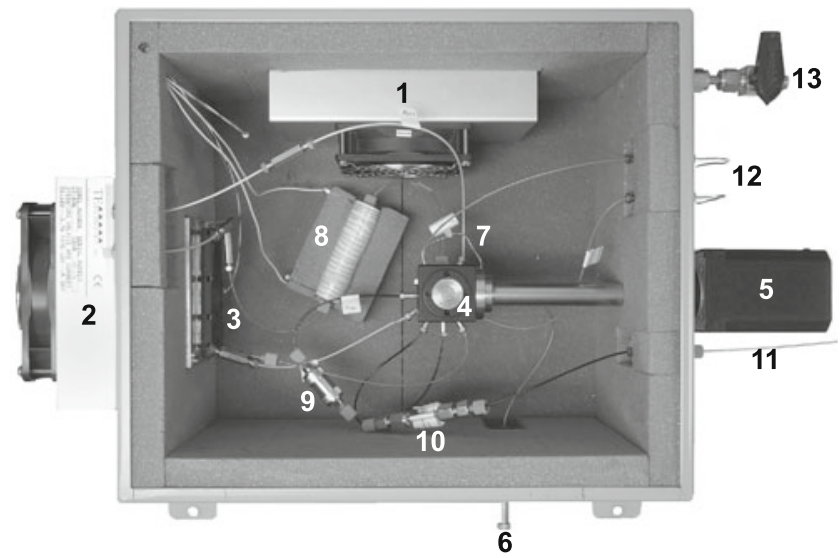

Digest and trap
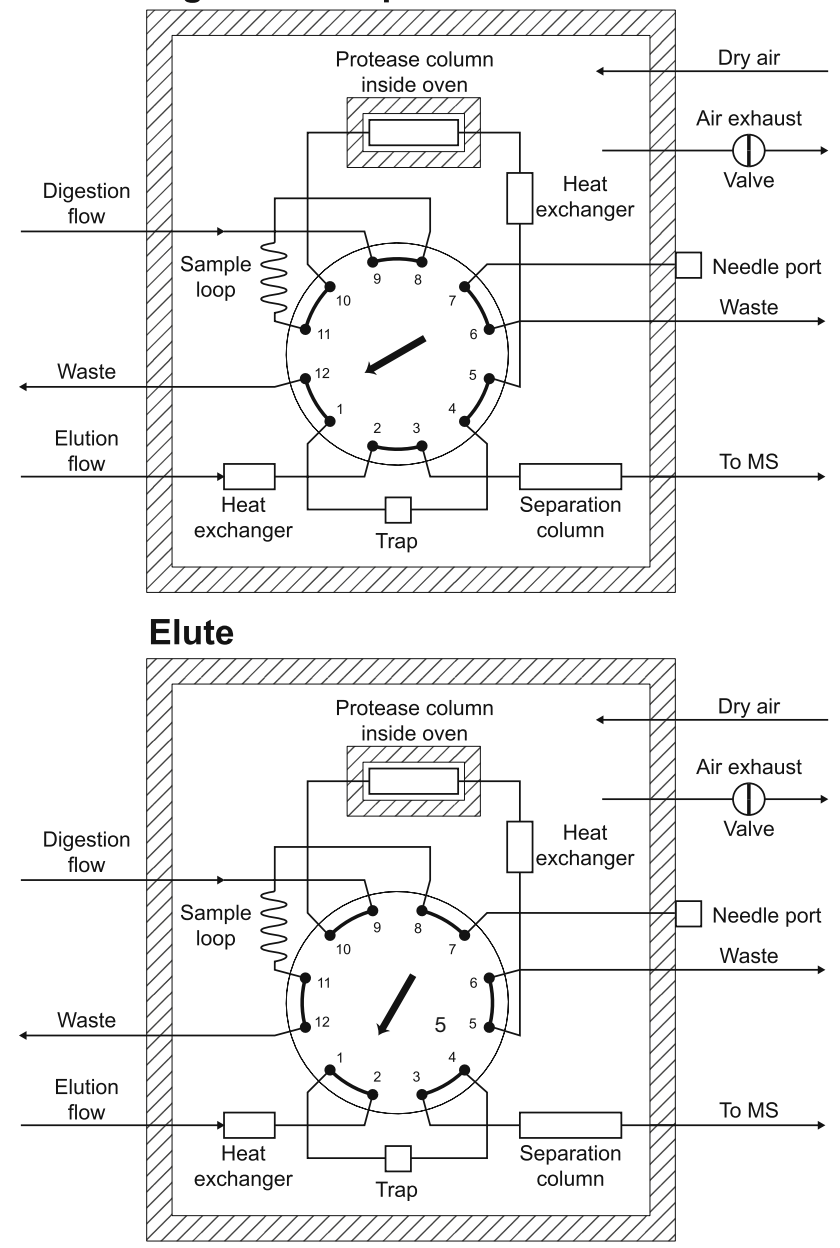

regulated by independent proportional integral derivative (PID) temperature controllers (see Figure S1). One innovative feature of our system is immediate cooling of peptides back to $0{ }^{\circ} \mathrm{C}$ at the outlet of the protease column oven (see Fig. 1). The efficiency of the heat exchangers increases with increasing flow rate (see Figure S3 and Table S1). By setting the heat exchangers to $-4{ }^{\circ} \mathrm{C}$, the mobile phase cools to at least $0{ }^{\circ} \mathrm{C}$ at all flow rates. The unit was fabricated almost entirely from offthe-shelf assemblies. The total cost of materials was less than US $\$ 5000$. Assembly required only simple machining of metal
Figure 1. Liquid handling in the refrigerated system. The top panel is a photograph of the refrigerated system viewed from above with the lid open. The labeled parts are (1) thermoelectric air cooler module, (2) thermoelectric cold plate module, (3) mobile phase heat exchangers, (4) twelve port switching valve, (5) valve actuator, (6) needle port, (7) sample loop, (8) protease column inside oven, (9) peptide trap, (10) peptide separation column, (11) MS inlet line, (12) waste lines, and (13) inlet and outlet for dry air purge. For visual presentation, the cover of the protease column oven (8) has been removed. The temperature controllers are not shown. The image was processed using Photoshop (Adobe Systems Inc., San Jose, CA, USA) to correct for lens distortion, to remove glare spots, and to remove the background, cover, and some wiring. The lower panels show schematic diagrams of the flow paths with the switching valve in the digest and trap position (middle) and the elute position (bottom). For clarity, not all components are oriented as shown in the photograph. Further details are provided in the Supplemental Materials

parts and hand cutting of foam insulation. Individuals interested in obtaining additional information should contact the corresponding author.

The chromatographic system consists of two pumps and online digestion using an immobilized protease column [9], but we use a single 12-port valve to handle the entire analysis (see Fig. 1). Simple contact closures programmed in the gradient time-table trigger valve actuation (see Figure S2). The loop is manually loaded with the valve in the elute position (Fig. 1, lower panel). When triggered by an external push-button controller, the valve switches to the digest and trap position (Fig. 1, middle panel). The digestion flow ( $0.1 \%$ formic acid) carries the sample through the loop and protease column. The resultant peptides are trapped, concentrated, and desalted on a reversed-phase peptide trap. (Our system is readily converted to analysis of intact proteins by replacing the protease column with a union.) When the valve is switched back to the elute position, a water/acetonitrile gradient elutes the peptides from the trap and separates them on a reversed-phase column. Simultaneously, the protease column is flushed to waste. By forming the gradient outside the housing, our refrigerated system is readily interfaced with any gradient HPLC system without re-plumbing. In particular, our capillary HPLC pump includes a mixer, pulse damper, proportioning valve, and flow sensor that remain under ambient conditions. Although our system is not compatible with 600 bar pressures, simple upgrades would enable UHPLC operation.

\section{Temperature Stability}

We measured air temperature in the enclosure and the mobile phase temperature (water, $200 \mu \mathrm{L} \mathrm{min}{ }^{-1}$ ) over a period of nine hours from a room temperature start. The system reached a stable operating temperature of $\sim 0^{\circ} \mathrm{C}$ in less than one hour (see Fig. 2). With the protease column oven set to $15^{\circ} \mathrm{C}$, the enclosure air temperature was stable at $0.5 \pm 0.1^{\circ} \mathrm{C}$. (With the protease column 
oven turned off, the enclosure temperature was $-0.1 \pm 0.1{ }^{\circ} \mathrm{C}$.) We evaluated the stability of the fluid temperature under several different scenarios. Water exiting the heat-exchanger positioned at the outlet of an unheated protease column (see Fig. 1) was $-2.2 \pm 0.1{ }^{\circ} \mathrm{C}$ (see Fig. 2, Heater Off). With the protease column oven heated to $15{ }^{\circ} \mathrm{C}$, the protease column effluent remained near $13.0{ }^{\circ} \mathrm{C}$ (Fig. 2, Protease Out), demonstrating both its stability and efficiency. Heating the protease column increases digestion efficiency, but would accelerate back-exchange. A heat exchanger located immediately downstream from the protease column cools the effluent back to $-2.2 \pm 0.1{ }^{\circ} \mathrm{C}$ (Fig. 2,

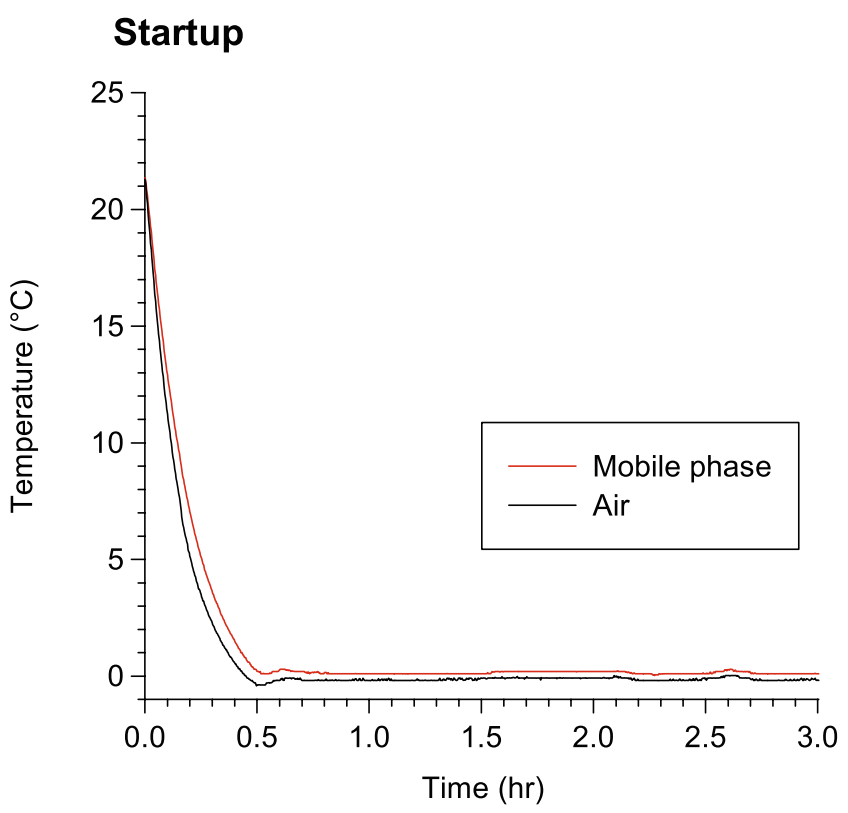

\section{Enclosure}

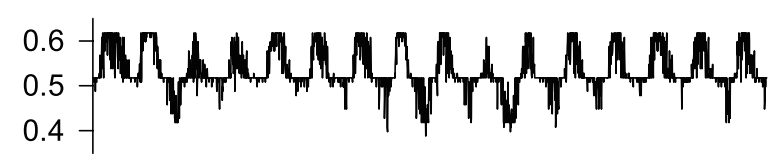

Heater Off

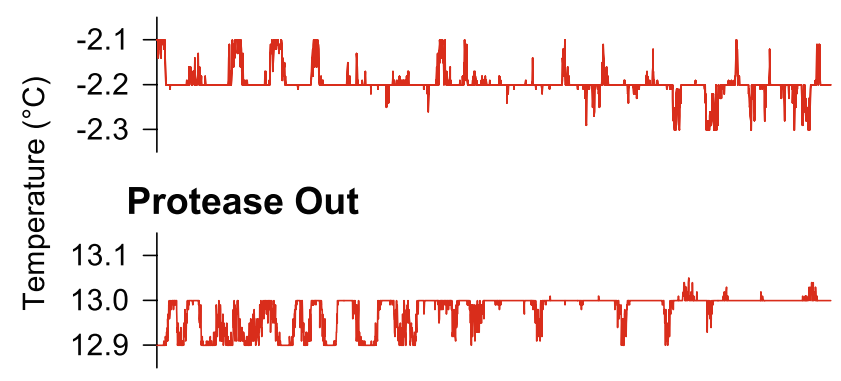

\section{Exchanger Out}

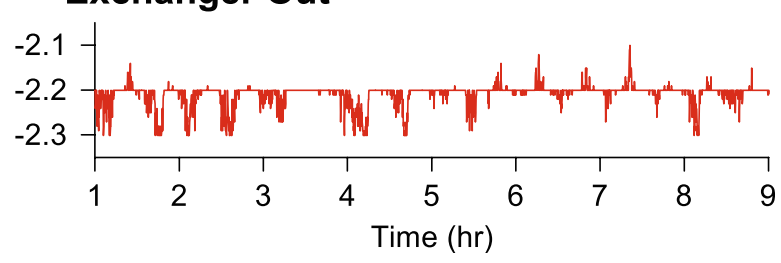

Exchanger Out). The rapid but small fluctuations are on the order of the precision limit of our temperature meters. Under quench conditions ( $\mathrm{pH} 2.5)$, these fluctuations would result in approximately a $1 \%$ decrease in the half-life of amide $\mathrm{D} / \mathrm{H}$ backexchange $[10,11]$, hence this degree of temperature stability is suitable for carrying out H/D-MS.

The components approach thermal equilibrium with the air, held at $0{ }^{\circ} \mathrm{C}$. We found that supercooling of the water did not persist beyond the outlet of the heat exchanger: the temperature of water flowing at $200 \mu \mathrm{L} \mathrm{min}^{-1}$ as it exited port \#6 (see Fig. 1) had increased to $0.1{ }^{\circ} \mathrm{C}$. The modest, transient supercooling of water in the absence of ice-forming nuclei is a well-studied phenomenon with literature dating back almost 200 y [12]. For example, NMR spectra of proteins in supercooled liquid water at $-15{ }^{\circ} \mathrm{C}$ were acquired from samples in $1.0 \mathrm{~mm}$ diameter glass capillaries [13]. Given the narrow dimensions of our tubing $(<200 \mu \mathrm{m})$ and the short residence time of the fluid in the heat exchanger, supercooling is not surprising.

\section{Chromatographic Performance}

We assessed chromatographic performance under refrigerated conditions using an optimized water/acetonitrile gradient and a column with superficially porous $\mathrm{C} 18$ particles. For complex digests, chromatographic resolution is critical for minimizing MS overlap and ion suppression. For the myoglobin digest, peak widths at half maximum ranged from 3.6 to $7.5 \mathrm{~s}$, with a median of $4.4 \mathrm{~s}$ (see Table 1 and Figure S4). For comparison, the median peak width obtained using UHPLC for the room temperature separation of a phosphorylase B digest was $2.7 \mathrm{~s}$ [6]. Hence the chromatographic performance remains commendable. In addition, since our system does not operate at the high system pressures required in UHPLC, we minimize the potential of extra back-exchange caused by frictional heating in the column [14].

Figure 2. Refrigeration of mobile phases and enclosure air from the initial startup is rapid and temperatures are stable once set points are reached. The top panel (Startup) shows the amount of time required for both temperatures to reach their respective set points. Here, the water temperature was measured at the inlet of the sample loop. The lower panels show temperature stability after the set points had been reached. (Enclosure) shows the air temperature with the protease column oven operating at a set point of $15{ }^{\circ} \mathrm{C}$. (Heater Off) shows the temperature of water emerging from the heat exchanger immediately downstream of the protease column with the column oven turned off. (Protease Out) shows the temperature of water emerging from the protease column operating at a set point of $15^{\circ} \mathrm{C}$. (Exchanger Out) shows the temperature of water after being cooled by the post-oven heat exchanger. The flow rate for all measurements was $200 \mu \mathrm{L} \mathrm{min}^{-1}$. Temperature measurements were started when the cooling system was turned on at $t=0 \mathrm{~h}$ 


\section{Deuterium Recovery}

For fully deuterated myoglobin peptides, the deuterium recovery ranged from $63.2 \%$ to $97.7 \%$ with a mean of $80.2 \%$ (Table 1 and Figure S5). Back-exchange calculations (at $\mathrm{pH}$ $\left.2.5,0{ }^{\circ} \mathrm{C}\right)[10,11]$ show that the wide range of deuterium recoveries is not surprising. For example, for peptide 110-134, which has a calculated half-life of $46 \mathrm{~min}$, we measured $63.2 \%$ deuterium recovery, while for peptide $8-13$, which has a halflife of $128 \mathrm{~min}$, we measured $97.7 \%$ deuterium recovery. Heating the protease column from $0{ }^{\circ} \mathrm{C}$ to $15{ }^{\circ} \mathrm{C}$ resulted in a slight decrease in deuterium recovery, from $71.7 \%$ to $70.5 \%$, with fully deuterated angiotensin I (see Table 1).

Our deuterium recovery results are at least as good as recovery obtained using other systems. An average of $70.0 \%$ recovery for cytochrome $c$ peptides was obtained with a UHPLC system [6]. With an ultrafast elution gradient, 78\% recovery for the test peptide LHRH was obtained [15]. Recovery using an HPLC system in a $0{ }^{\circ} \mathrm{C}$ water bath measured using angiotensin I that had been labeled for over 1 mo [4] appears to be $\sim 84 \%$ ( $\sim 5.9 \mathrm{Da}$ increase), but when we account for slow $\mathrm{H} / \mathrm{D}$ exchange at the $\mathrm{C} 2$ carbons of the imidazole rings of two histidine residues [16], the amide deuterium recovery was $\sim 56 \%$. Hence, our recovery of $71.7 \%$ for this same peptide, labeled for only $1-2 \mathrm{~h}$, was considerably higher. In addition to excellent recovery, our system demonstrated outstanding reproducibility, with a mean relative standard deviation of $\pm 0.91 \%$ for measured deuterium uptake in myoglobin peptides (Table 1). For the ultrafast elution gradient of test peptide LHRH, $0.71 \%$ error was reported in deuterium uptake measurements [15]. While these results may not be directly comparable because of differences between methods, deuterium recovery using our refrigerated system and a typical gradient is more than satisfactory for H/D-MS analyses.

\section{Conclusions}

We have constructed an inexpensive refrigerated system and evaluated its performance for H/D-MS. The temperatures of the mobile phases were directly measured to validate the effectiveness of cooling, something that has not been previously reported. Small volume heat exchangers efficiently cooled the mobile phases, allowing gradients to be formed at room temperature. This efficiency eliminates the need for large dwell volume for cooling that can delay or broaden gradients, a significant advantage when many samples need to be run in succession. Efficient and stable cooling of the system resulted in excellent deuterium recovery and reproducibility. Use of a superficially porous reversed-phase column gave excellent chromatographic results with short gradients under quenched conditions $\left(\mathrm{pH} 2.5,0{ }^{\circ} \mathrm{C}\right)$ without the need for ultra-high pressures.
Finally, our refrigerated LC system can be readily interfaced with any LC/MS system since only simple contact closures are required to drive valve actuation.

\section{Acknowledgments}

The authors acknowledge financial support for this project provided by the University of Kansas. The authors thank David Scott for efforts to develop a prototype system based on a domestic refrigerator. Anthony Ziegler and Andrew Gieschen of Agilent Technologies provided technical advice on heat exchangers. Allan Hase, Danny Michael, Ash Shadrick, and Jeff Worth contributed to construction.

\section{References}

1. Konermann, L., Tong, X., Pan, Y.: Protein structure and dynamics studied by mass spectrometry: H/D exchange, hydroxyl radical labeling, and related approaches. J. Mass Spectrom. 43, 1021-1036 (2008)

2. Rosa, J.J., Richards, F.M.: An experimental procedure for increasing the structural resolution of chemical hydrogen-exchange measurements on proteins: Application to ribonuclease S peptide. J. Mol. Biol. 133, 399-416 (1979)

3. Zhang, Z., Smith, D.L.: Determination of amide hydrogen exchange by mass spectrometry: A new tool for protein structure elucidation. Protein Sci. 2, 522-531 (1993)

4. Burkitt, W., O'connor, G.: Assessment of the repeatability and reproducibility of hydrogen/deuterium exchange mass spectrometry measurements. Rapid Commun. Mass Spectrom. 22, 3893-3901 (2008)

5. Chalmers, M.J., Busby, S.A., Pascal, B.D., He, Y., Hendrickson, C.L., Marshall, A.G., Griffin, P.R.: Probing protein ligand interactions by automated hydrogen/deuterium exchange mass spectrometry. Anal. Chem. 78, 1005-1014 (2006)

6. Wales, T.E., Fadgen, K.E., Gerhardt, G.C., Engen, J.R.: High-speed and high-resolution UPLC separation at zero degrees celsius. Anal. Chem. 80, 6815-6820 (2008)

7. Weis, D.D., Engen, J.R.: Microflow HPLC/ESI enhances hydrogen exchange mass spectrometry. Protein Sci. 14(Suppl 1), 213 (2005)

8. Resing, K.A., Ahn, N.G.: Deuterium exchange mass spectrometry as a probe of protein kinase activation. Analysis of wild-type and constitutively active mutants of MAP kinase kinase-1. Biochemistry 37, 463-475 (1998)

9. Wang, L., Pan, H., Smith, D.L.: Hydrogen exchange-mass spectrometry: optimization of digestion conditions. Mol. Cell. Proteom. 1, 132-138 (2002)

10. Bai, Y., Milne, J.S., Mayne, L., Englander, S.W.: Primary structure effects on peptide group hydrogen exchange. Proteins 17, 75-86 (1993)

11. Connelly, G.P., Bai, Y., Jeng, M.-F., Englander, S.W.: Isotope effects in peptide group hydrogen exchange. Proteins 17, 87-92 (1993)

12. Angell, C.A.: Supercooled water. Annu. Rev. Phys. Chem. 34, 593-630 (1983)

13. Skalicky, J.J., Mills, J.L., Sharma, S., Szyperski, T.: Aromatic ringflipping in supercooled water: implications for NMR-based structural biology of proteins. J. Am. Chem. Soc. 123, 388-397 (2001)

14. Gritti, F., Martin, M., Guiochon, G.: Influence of viscous friction heating on the efficiency of columns operated under very high pressures. Anal. Chem. 81, 3365-3384 (2009)

15. Zhang, H.-M., Bou-Assaf, G.M., Emmett, M.R., Marshall, A.G.: Fast reversed-phase liquid chromatography to reduce back exchange and increase throughput in H/D exchange monitored by FT-ICR mass spectrometry. J. Am. Soc. Mass. Spectrom. 20, 520-524 (2009)

16. Miyagi, M., Nakazawa, T.: Determination of $\mathrm{pKa}$ values of individual histidine residues in proteins using mass spectrometry. Anal. Chem. 80, 6481-6487 (2008) 